\title{
Cadmio: Biacumulación y efecto en el desarrollo vegetativo de haba (Vicia faba L.) como propuesta de bioensayo
}

Cadmium: Biacumulation and effect on the vegetative development of broad bean (Vicia faba L.) as a bioassay proposal

Sánchez Zepeda María Yesenia ${ }^{1}$, Alberto José Gordillo Martínez ${ }^{2 凶}$, Maritza López Herrera ${ }^{1}$, Francisco Prieto García ${ }^{2}$, Juan Carlos Gaytán Oyarzún ${ }^{1}$

${ }^{1}$ Área Académica de Biología, ${ }^{2}$ Área Académica de Química, Universidad Autónoma del Estado de Hidalgo

${ }^{\bowtie}$ Autor para correspondencia: gordillo@uaeh.edu.mx

Aceptado: 15/11/2018

\section{RESUMEN}

Se evaluó el efecto del cadmio en el crecimiento y ganancia de biomasa en plantas de haba (Vicia faba L.), desarrolladas en cultivo hidropónico y expuestas a una concentración de $\mathrm{CdSO}_{4}$ de 0.112 ppm; se determinó también la bioacumulación del metal en raíz, tallo y hoja. Los resultados muestran que el cadmio generó una disminución en el crecimiento de la parte vegetativa, en la longitud de la raíz y en la producción de biomasa en las plantas sometidas al contaminante; la raíz fue el sitio de mayor bioacumulación seguido de las hojas y el tallo. Las raíces mostraron un oscurecimiento y engrosamiento. Las respuestas al contaminante pueden ser una herramienta para estudios de monitoreo ambiental.

Palabras clave: Raíz, tallo, biomasa.

\section{ABSTRACT}

he effect of cadmium on the growth and biomass gain in bean plants (Vicia faba L.), developed in hydroponic culture and exposed to a $\mathrm{CdSO}_{4}$ concentration of $0.112 \mathrm{ppm}$, was evaluated; The bioaccumulation of the metal in root, stem and leaf was also determined. The results show that the cadmium generated a decrease in the growth of the vegetative part, in the length of the root and in the production of biomass in the plants subjected to the contaminant; the root was the site of greatest bioaccumulation followed by the leaves and the stem. The roots showed a darkening and thickening. Responses to the pollutant can be a tool for environmental monitoring studies.

Keywords: Root, stem, biomass.

\section{INTRODUCCIÓN}

Los metales pesados contribuyen de una manera importante a la contaminación ambiental (Nedelkoska y Doran, 2000); algunos de ellos como el $\mathrm{Cu}$ y el $\mathrm{Zn}$ son esenciales para las plantas al ser necesarios para su crecimiento y desarrollo, sin embargo, una alta concentración de tanto de los elementos esenciales como los no esenciales pueden generar un efecto tóxico y promover la inhibición del crecimiento en la mayoría de las plantas (Hall, 2002). 
El cadmio (Cd) es un elemento no esencial que se libera al ambiente por actividades humanas como la fertilización con compuestos fosfatados, la eliminación de desechos domésticos, municipales e industriales. Estas fuentes pueden generar un incremento del $\mathrm{Cd}$ en el suelo y por lo consiguiente un incremento del metal en los cultivos. Es un contaminante altamente peligroso debido a su alta toxicidad y gran solubilidad en el agua (Jiang et al., 2001).

Los efectos fitotóxicos de los metales pesados en plantas se manifiestan entre otros con una reducción en el crecimiento y la acumulación de biomasa. Se tienen evidencias de que el $\mathrm{Cd}$ inhibe el crecimiento de las raíces y la división celular en diferentes plantas (Steinkellner et al., 1998; Fojtova y Kovarik, 2000; Yi y Meng, 2003).

El objetivo de este proyecto fue evaluar la bioacumulación y el efecto del cadmio en el crecimiento y ganancia de biomasa en plantas de haba (Vicia faba L.) var. Major.

Materiales y métodos

Material vegetal

Se utilizaron semillas certificadas de haba (Vicia faba L.) var. Major, mismas que se conservaron en un lugar fresco y seco hasta el inicio de la experimentación.

\section{MATERIALES Y MÉTODOS}

Las semillas se germinaron bajo condiciones controladas de laboratorio $\left(25 \pm 1^{\circ} \mathrm{C}\right.$, y en obscuridad), cuando la radícula alcanzó $1 \mathrm{~cm}$ de longitud, se trasplantaron a un sustrato inerte (peatmost, vermiculta y agrolita, 1:1:1) y fueron regadas con solución nutritiva comercial hasta que alcanzaron una longitud del tallo entre $15 \mathrm{y}$ $20 \mathrm{~cm}$; las plántulas se trasplantaron a un sistema hidropónico con solución nutritiva comercial; se usaron dos contenedores $(80 \mathrm{~cm}$ x $45 \mathrm{~cm}$ x 25 cm), que contenían 23 lt de solución; un contenedor se mantuvo solamente con la solución nutritiva (Testigo) y al otro se le agregaron $92 \square \mathrm{L}$ de una solución stock de $\mathrm{CdSO}_{4}$ para generar una solución de $0.112 \mathrm{ppm}$ de $\mathrm{CdSO}_{4}$, valor límite establecido por la Norma Oficial Mexicana (NOM-001-ECOL-1996). Las plantas se mantuvieron en estas condiciones por siete semanas, y al término de este periodo, se midió la longitud final del tallo, hojas y raíces de las plantas, finalmente se separaron en sus diferentes componentes (tallo, hoja y raíz). Las muestras se secaron en una estufa a $70^{\circ} \mathrm{C}$ por 72 $\mathrm{hr}$, de esta forma se obtuvo el peso seco; este material se utilizó para determinar la bioacumulación de $\mathrm{Cd}$ en cada órgano de la planta.

Bioacumulación.

La determinación del porcentaje de bioacumulación del Cd se realizó por la técnica de Espectrofotometría de Absorción Atómica de Flama (marca Perkin Elmer modelo PinAAcle 900T), se empleó una lámpara Cd y con una longitud de onda de $228.8 \mathrm{~nm}$. Las determinaciones se realizaron en Laboratorio de Análisis y Monitoreo Ambiental del CIIEMADIPN (Centro Interdisciplinario de Investigaciones y Estudios sobre Medio Ambiente y Desarrollo del Instituto Politécnico Nacional).

\section{Diseño experimental y estadístico}

Se utilizó un diseño completamente al azar con dos muestras (Testigo y solución de 0.112 ppp de $\mathrm{CdSO}^{4}$ ) con tres repeticiones cada una. Los datos se analizaron mediante el programa estadístico Past 3.2. Se probó la normalidad de los datos con la prueba de Shapiro-Wilk y se realizó un ANOVA de una vía y una comparación de medias Tukey con una $\mathrm{P}<0,05$. 


\section{RESULTADOS}

El análisis estadístico muestra diferencias significativas $(\mathrm{P}<0.001)$ en el contenido de $\mathrm{Cd}$ en los diferentes órganos de las plantas sometidas a $\mathrm{CdSO}_{4}$. Se determinó que el $\mathrm{Cd}$, se bioacumula principalmente en la raíz de la planta, seguida de las hojas y el tallo respectivamente, en el caso del control, las plantas presentaron bioacumulación de $\mathrm{Cd}$ en muy bajas concentraciones en los tres órganos evaluados raíz, tallo y hojas respectivamente $(1.64 \mathrm{mg} / \mathrm{kg}, 0.59 \mathrm{mg} / \mathrm{kg}$ y $0.09 \mathrm{mg} / \mathrm{kg})$, la raíz fue el órgano en donde esta bioacumulación fue mayor, sin embargo, estadísticamente no hay diferencias entre ellos ( $\mathrm{P}=0.796)$ (Figura 1). Es evidente que las plantas sometidas al metal

Por otro lado, los resultados referentes al crecimiento de las plantas y la cuantificación del PS, muestran que las plantas sometidas al Cd, presentaron una reducción importante en el crecimiento y la ganancia de biomasa por efecto del metal. El análisis estadístico muestra diferencias significativas $(\mathrm{P}<0.001)$ (Figura $2 \mathrm{~A}$ y B).

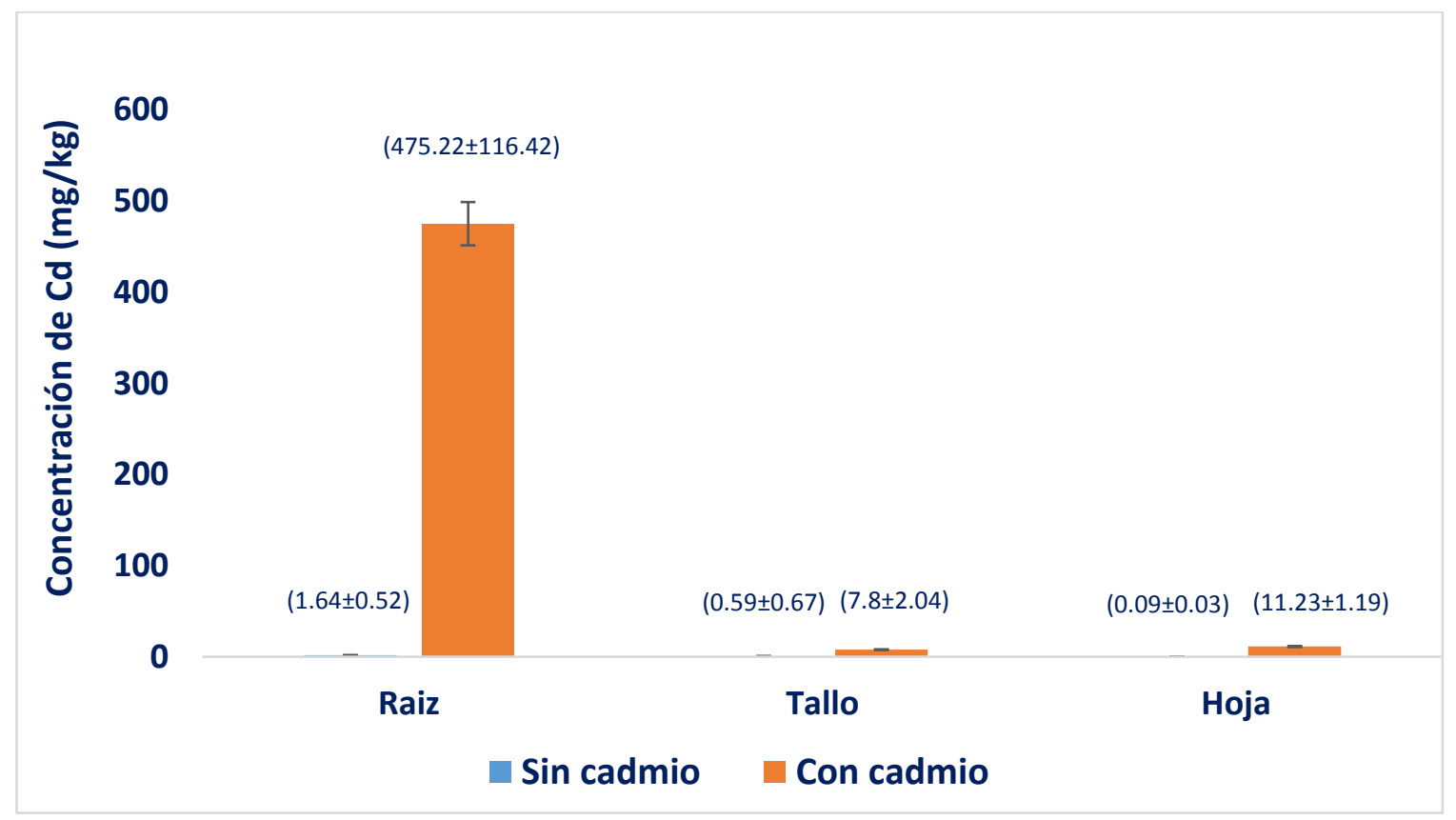

Figura 1. Bioacumulación de $\mathrm{Cd}$, en los diferentes órganos (tallo, hojas y raíces) de plantas de Vicia faba L., sometidas a dos concentraciones de $\mathrm{CdSO}_{4}(0.0 \mathrm{ppm}$ y $0.112 \mathrm{ppm})$ durante siete semanas. 

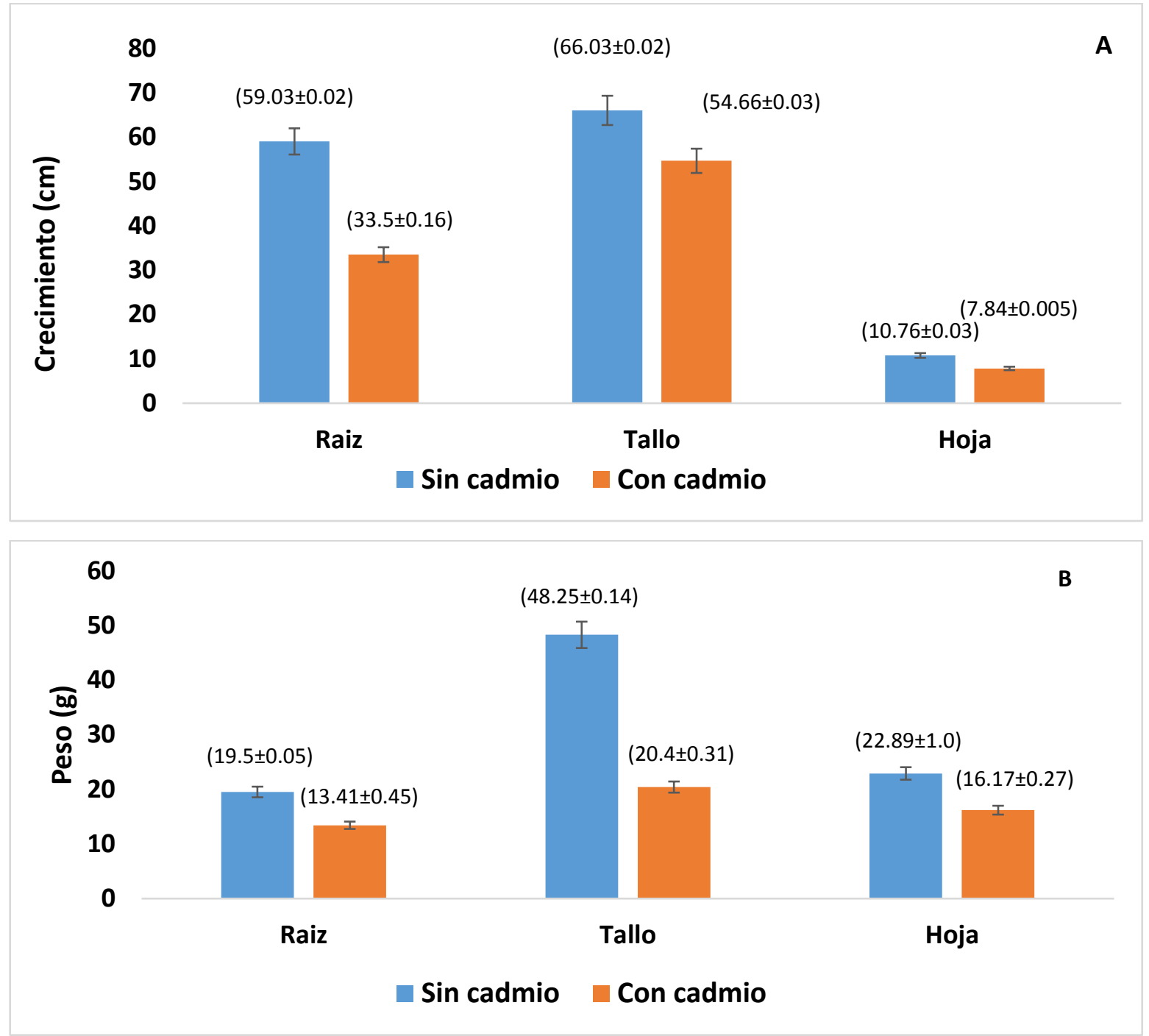

Figura 2. Longitud final de tallo, hojas y raíz (A) y ganancia de biomasa (B) de plantas de Vicia faba L. sometidas a dos concentraciones de $\mathrm{CdSO}_{4}(0.0 \mathrm{ppm}$ y $0.112 \mathrm{ppm})$.

Las plantas expuestas al tratamiento de contaminación desarrollaron síntomas visuales en respuesta a la toxicidad por metales pesados. Las raíces de las plantas sometidas al $\mathrm{Cd}$, presentaban un oscurecimiento y engrosamiento en comparación con las del control (Figura 3).

\section{DISCUSIÓN}

De manera general los resultados de esta investigación muestran que las plantas sometidas al cadmio presentaron un menor crecimiento tanto de la porción vegetativa como de la raíz, dando como resultado una menor ganancia de biomasa. Diversas investigaciones muestran que la presencia del $\mathrm{Cd}$ en el medio de crecimiento de las plantas, tiende a disminuir la longitud de las raíces y la biomasa total de la planta, además de afectar otras actividades metabólicas (Dixit et al., 2001; Pedrosa et al., 2011; García-Gallegos et al., 2012; MéndezHurtado et al., 2013). 


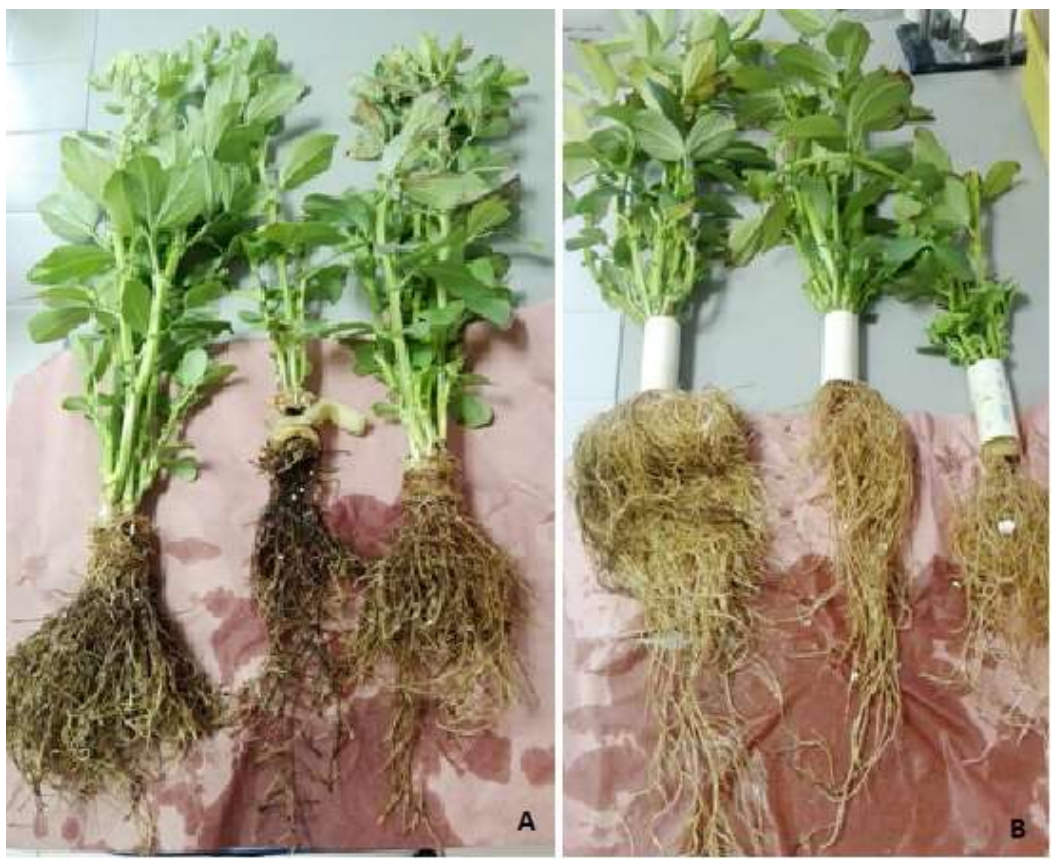

Figura 3. Síntomas del efecto del Cd en la raíz de haba ( $V$. faba L.) sometida a 0.112 ppm (A) y 0.0 ppm (B) de $\mathrm{CdSO}_{4}$.

Los resultados del análisis de bioacumulación indica que la mayor parte del cadmio quedó bioacumulado en las raíces de las plantas, seguida las hojas y finalmente el tallo. El cadmio analizado se encontró tanto en las plantas sometidas a cadmio como en las que no se sometieron a él; el $\mathrm{Cd}$, aun cuando no es un elemento esencial, puede encontrarse en la planta en bajas concentraciones $\left(<1 \mathrm{mg} \mathrm{kg}^{-1}\right)$, siendo fácilmente absorbido y acumulado por las raíces, limitando su transporte a la parte aérea (Almagro et al., 2015).

El oscurecimiento y engrosamiento de las raíces por efecto de la contaminación por metales es una sintomatología que se ha reportado en otras investigaciones (Pedrosa et al., 2011). Se cree que esta sintomatología podría justificar de alguna forma, la menor producción de biomasa, ya que el Cd también interfiere en la captura, transporte y uso de varios micronutrientes como el Fe, Mn y Zn (Mendez-Hurtado et al., 2013). La toxicidad resultado como resultado de la unión de los iones metálicos a grupos sulfhidrilos de las proteínas generando una inhibición de la actividad o la alteración de la estructura o el desplazamiento de un elemento esencial generándose una deficiencia en las plantas (van Assche y Clijsters, 1990), esto generaría un menor crecimiento, aunado a esto, se sabe que el $\mathrm{Cd}$, puede llegar a sustituir el átomo de $\mathrm{Mg}$, que forma parte de la molécula de clorofila, trayendo como consecuencia una disminución en la actividad fotosintética afectando la producción de biomasa de las plantas expuestas al contaminante (He et al., 2008). 


\section{CONCLUSIONES}

La presencia de Cd afectó el crecimiento de la raíz y la parte vegetativa de las plantas de haba, provocando una disminución en la ganancia de biomasa total. La raíz fue el órgano en donde se acumuló la mayor parte del cadmio seguido de hojas y tallo, como resultado de esto, las raíces presentaron una alteración morfológica, adquiriendo una coloración oscura y un engrosamiento del tejido, lo cual puede suponer que tuvo un efecto en la absorción de nutrientes generando un pobre desarrollo de las plantas sometidas al contaminante. Estas respuestas biológicas (crecimiento y sintomatología de la raíz) pueden ser buenos indicadores de toxicidad para plantas expuestas al $\mathrm{Cd}$, y ser propuestas como una herramienta para estudios de monitoreo ambiental.

\section{AGRADECIMIENTOS}

Un agradecimiento especial al Ing. Evanibaldo González Gómez, por su invaluable apoyo en los análisis de bioacumulación realizados en el CIIEMAD-IPN.

\section{LITERATURA CITADA}

Almagro, L., Segura-Reinaldos A. M., Pedreño M. A., Bernal M. P. 2015. Tolerancia y acumulación de metales pesados y As en diferentes especies de Cistus L. Anales de Biología 37: 143-153.

https://doi.org/10.6018/analesbio.37.16

Dixit, V., Vivek, P., Shyam, T. (2001).

Differential antioxidative responses to cadmium in roots and leaves of pea (Pisum sativum L. cv. Azad). Journal of Experimental Botany 52: 1101-1109. https://doi.org/10.1093/jexbot/52.358.110 Fojtova, M., Kovarik, A. 2000. Genotoxic effect of cadmium is associated with apoptotic changes in tobacco cells. Plant Cell Environmental 23:531-537.

https://doi.org/10.1046/j.1365-3040.2000. García E G García E Nánchez et al., 2018 Juárez, L.S., Montiel, J.M.R.G., Gómez, M.A.C. 2012. La respuesta de haba (Vicia faba L.) cultivada en un suelo contaminado con diferentes concentraciones de cadmio. Revista Internacional de Contaminación Ambiental 28 (2): 119-126.

Hall, J.L. 2002. Cellular mechanisms for heavy metal detoxification and tolerance. Journal of Experimental Botany 53: 1-11. https://doi.org/10.1093/jxb/53.366.1

He, J.Y., Ren, Y.F., Hu, C.Z., Yan, Y. P., Jiang, D.A. 2008. Effect of $\mathrm{Cd}$ on growth, photosynthetic gas exchange, and chlorophyll fluorescence of wild and Cdsensitive mutant rice. Photosynthetica 46: 466-470. https://doi.org/10.1007/s11099-0 Jiang, W.; Liu, D., Hou, W. 2001. Hyperaccumulation of cadmium by roots, bulbs and shoots of garlic (Allium sativum L.). Bioresource Technology 76: -13. https://doi.org/10.1016/S0960-8524(00)00 Mendez-Hurtado, A., Rangel-Mendez, R., Yáñez-Espinoza, L., Flores, J. 2013. Tolerance cadmium of agave lechuguilla (Agavaceae) seeds and seedlings from sites contaminated with heavy metals. The Scientific World Journal. Article ID 167834, $11 \quad$ pages. https://doi.org/10.1155/2013/167834

Nedelkoska, T.V., Doran, P.M. 2000. Characteristics of heavy metal uptake by plants species with potential for phytoremediation and phytomining. Minerals Engineering 13: 549-561.

https://doi.org/10.1016/S0892-6875(00)00 Norma Oficial Mexicana (NOM-001-ECOL1996). Establece los límites máximos permisibles de contaminantes en las descargas de aguas residuales en aguas y bienes nacionales. Secretaría de Medio Ambiente, Recursos Naturales, DOF 06/01/1997. Página en red: http://dof.gob.mx/nota_detalle.php?codig 
$\mathrm{o}=4863829 \&$ fecha $=06 / 01 / 1997$. Consultado: 25 de febrero 2018.

Pedrosa, G. M., Lara Lanza de Sá e Melo Marques, T. C., Goncales, N. M de O., De Castro, E. M., Soares, A. M. 2011. Ecophysiological and anatomical changes due to uptake and accumulation of heavy metal in Brachiria decumbens. Science Agricola 68(5) p. 566-573.

https://doi.org/10.1590/S0103-901620110

Steinkellner, H., Mun-Sik, K., Helma, C., Ecker,
S., Te-Hsiu, M., Horak, O., Kundi, M., Knasmüller, S. 1998. Genotoxic effects of heavy metals: comparative investigation with plant bioassay. Environmental Molecular Mutagenic 31: 183-191. https://doi.org/10.1002/(SICI)1098-2280(1 Yi, H., Meng, Z. 2003. Genotoxicity of hydrated sulfur dioxide on root tips of Allium sativum and Vicia faba. Mutation Research 537: 109-114.

https://doi.org/10.1016/S1383-5718(03)00 